\title{
Quelques propositions quant aux Sonnets de Shakespeare
}

\section{Yves Bonnefoy}

\section{OpenEdition}

\section{Journals}

Édition électronique

URL : http://journals.openedition.org/shakespeare/1020

DOI : 10.4000/shakespeare.1020

ISSN : 2271-6424

\section{Éditeur}

Société Française Shakespeare

\section{Édition imprimée}

Date de publication : 1 novembre 2007

Pagination : 13-38

ISBN : 2-9521475-3-1

\section{Référence électronique}

Yves Bonnefoy, «Quelques propositions quant aux Sonnets de Shakespeare », Actes des congrès de la Société française Shakespeare [En ligne], 24 | 2007, mis en ligne le 30 mars 2010, consulté le 01 mai 2019. URL : http://journals.openedition.org/shakespeare/1020 ; DOI : 10.4000/shakespeare.1020

Ce document a été généré automatiquement le 1 mai 2019

(C) SFS 


\title{
Quelques propositions quant aux Sonnets de Shakespeare
}

\author{
Yves Bonnefoy
}

\section{I}

1 D'abord, merci à Yves Peyré de son invitation à prendre la parole en ouverture de cet ouvrage. Je vois quelque sens à cela, car la sorte de propos que j'ai à tenir me garde en marge des travaux des chercheurs, qui tendent à l'acquisition de faits précis et à des analyses critiques en accord aussi poussé que possible avec ces derniers.

2 Il ne s'agit pas là, entre eux et moi, d'une différence de préoccupations, toutefois; ni de ma part d'un quelconque - et qui serait bien frivole - dédain pour la recherche historique, car nous avons le même projet, qui est de comprendre Shakespeare et son époque, et je ne conçois pas que je puisse m'attacher à ces grands problèmes sans vouloir profiter de leur savoir. Mais mon point de vue sur eux est distinct du leur, et ma façon de les aborder ne peut donc elle aussi qu'en différer.

3 Mon point de vue ? Je m'intéresse moins à Shakespeare comme tel qu'à ce qu'est la poésie, considérée par moi, à tort ou à raison, comme un des aspects essentiels, c'est-à-dire constants, à travers l'histoire, de la relation au monde de l'être parlant que nous sommes : de ce regard en proie au langage. La poésie comme une façon d'être, ou de vouloir être, et non pas un texte.

4 Et ma méthode, si ce mot n'est pas prétentieux - on peut le penser -, sera de tenter de percevoir et d'identifier ce qui est poésie, spécifiquement, dans la complexité et l'ambiguïté d'un texte : celui-ci poème souvent mais aussi bien récit ou même, comme dans le cas de Shakespeare, pièce de théâtre. Reconnaître le poétique là où il est dans ces créations de diverses sortes, soit en le retrouvant comme leur motivation, soit par ses effets, directs ou indirects, sur leur élaboration par leur auteur ou d'autres poursuites de ce poète, soit même dans une réflexion de ce dernier, consciente ou pas, sur la poésie 
dans son travail même. Aucune œuvre n'est seulement poésie. J'ai en vue une analyse spectrale qui en isolerait le rayon parmi des réfractions différentes.

Et, certes, je viens déjà de le reconnaître, rien n'apparaîtra de sérieux dans cette analyse si elle n'est pas conduite avec en esprit le contexte de l'œuvre, autrement dit sa langue d'abord, en son vocabulaire et sa syntaxe d'époque, mais aussi les concepts, les catégories de pensée, les idées philosophiques ou les croyances, religieuses ou autres, qui ont caractérisé son moment, parfois très bref. Et c'est pour cela que je me préoccupe des travaux des chercheurs, non pour rivaliser avec eux mais pour accéder à ce qui m'importe. L'approche même philosophique des œuvres de poésie est tributaire de la recherche des historiens et des philologues. de penser que j'ai droit de recourir dans la lecture d'un poète - ou d'ailleurs aussi d'un grand peintre - à ce que j'aimerais appeler l'interprétation figurale, deux mots par lesquels j'entends l'analyse d'une pensée qui, dans la profondeur de l'écriture des œuvres, s'active tout autrement que la réflexion explicite, celle-ci beaucoup plus dépendante de la circonstance historique. Je crois, au moins dans le cas des grandes créations de l'esprit, à une pensée, celle-ci implicite ou même inconsciente, qui par déplacements ou condensations travaille là comme le rêve le fait, le rêve des nuits, et avec alors allégeance à d'autres préoccupations ou intuitions ou croyances que celles que l'on décèle dans l'époque ou même la langue contemporaines. En d'autres mots, il y a plusieurs niveaux de pensée dans tout ce qui est écriture, et le plus intérieur se situe au dessous de la détermination historique et des diverses lectures que celle-ci autorise.

7 Existe chez les écrivains ou peintres ou autres artistes une pensée "figurale ", de même façon que dans nos rêves. Et pourquoi je m'intéresse à cette pensée, c'est parce qu'elle a trait dans les œuvres de poésie à la poésie comme telle; et énonce, sur l'expérience qu'est celle-ci, des propositions à tout le moins méditables. Je suis prêt à conclure que c'est par cette voie du figural que l'on accède le mieux dans l'invention passée ou présente à ce qui caractérise la poésie. Il faut donc en prendre le risque.

Oui, c'est vrai, l'entreprise est aventureuse.

9 Mais qu'elle soit jugée à ses résultats, et au surcroît d'intérêt qu'elle peut valoir à certains aspects dans même des œuvres aussi étudiées qu'Hamlet ou les Sonnets de Shakespeare.

\section{II}

10 La poésie ? Je crains que beaucoup ne s'irritent de me voir en parler comme s'il s'agissait d'un invariant de l'esprit à travers les siècles.

11 Et si je crains cela, c'est parce que je n'ignore pas que les historiens du fait littéraire ou de la pensée aiment constater que l'idée de la poésie ou d'autres qui en sont proches varient avec évidence d'une époque à une autre, et souvent beaucoup. La façon dont le Moyen Âge comprenait le mot "poésie » n'a pas grand chose à voir avec les conceptions de la Renaissance ou du Romantisme. Une pensée du faire, de la production d'un objet verbal, une primauté accordée aux catégories et aux enseignements de la rhétorique, caractériserait tout un premier temps de ce mot qui plus tard évoquerait bien plutôt une "fureur» inspirée par la divinité puis une expérience aux limites de la mystique. Historien est qui sait cela. 
12 Mais comment ne pas constater quelques évidences? Et, par exemple, qu'Homère, ou Dante le médiéval, ou Ronsard le renaissant, ou Keats puis Baudelaire ou même Paul Valéry n'ont jamais cessé d'être tenus dès le premier jour pour semblablement et spécifiquement des poètes, en dépit des différences de leurs projets ou de leurs regards sur le monde? On les rapproche, instinctivement, pour une raison qui, encore que bien souvent mal comprise, les distingue en revanche de beaucoup d'autres sortes d'écrivains. Et d'ailleurs on voit bien qu'ils se reconnaissent entre eux. Ainsi lorsque Dante, au début de son grand poème, imagine qu'il rencontre Virgile, qui va lui servir de guide, et le salue en tant, précisément, que poète.

Or' sei tu quel Virgilio...

s'écrie Dante, avec une émotion qui montre bien que pour lui - et pour bien d'autres, n'en doutons pas - la poésie est tout autre chose que ce que calcule la rhétorique.

De ce fait je suis bien convaincu, moi aussi ; et je m'arrête un instant à cette question de la poésie. Il y a poésie depuis l'origine de la parole et ce fut toujours la même intuition, car la parole souffre d'une contradiction intérieure elle aussi toujours la même, et que les poètes tentent de dénouer ou à tout le moins de rendre plus supportable. Cette contradiction? C'est que pour penser et agir nous avons besoin de concepts, alors que ceux-ci ne se constituent qu'en prenant appui sur des aspects qu'ils séparent de la totalité de la chose, ce qui nous prive du plein de celle-ci au moment même où nous exerçons sur elle cette prise. Les concepts s'organisent en chaînes signifiantes qui substituent une appréhension de simples schèmes à la relation antérieure d'intimité à la chose : cette intimité qui nous gardait aussi au contact des autres êtres. Le monde s'est fait énigme, voilà ce que la poésie essaie de réparer, laquelle est donc née dès le second jour des mots, après quoi elle n'a plus cessé de tenter de nous rendre, au moins par instants, l'immédiateté perdue.

15 Une constante du fait humain, la poésie, aussi ancienne que la parole, et qui durera autant qu'elle.

16 Et comment ne pas se proposer de comprendre en quoi consiste au juste cet emploi second du langage et la façon dont il aborde les mots dont il entend restituer la plénitude, mais comment aussi ne pas vouloir suivre à travers les âges les changements qui l'ont affecté, par adaptation de sa constante intuition aux circonstances nouvelles de chaque époque ? Deux enquêtes qui ne sont d'ailleurs aucunement dissociables.

Sur la nature du poétique juste deux mots, pour introduire à mon souci d'aujourd'hui, la conscience qu'en eut Shakespeare.

Ce qu'est la poésie, c'est d'abord le sentiment que la moindre chose en sa finitude essentielle - c'est-à-dire en son être-là devant nous, ici, maintenant - est hors de portée de toute description ou autre saisie que voudrait en faire le simple discours conceptuel: elle lui est transcendante. Et la poésie éprouve d'instinct cette transcendance, elle ressent durement la condition d'exilés qui en résulte pour nous, mais elle dispose aussi d'un moyen, d'un grand moyen, pour tenter de reprendre pied dans l'immédiateté abolie, c'est le son qui est dans le mot. Car ce son, en tant que donnée sonore irréductible à tout sens, c'est à nouveau la plénitude de l'immédiat. Et faire vivre le son, par des rythmes et rimes ou assonances dans un emploi des vocables qui en devient un poème, ce pourra être décontenancer le discours conceptuel, c'est-à-dire lever un coin du voile.

19 Voici pourquoi, soit dit en passant, la poésie ne peut être considérée comme simplement la production d'un objet verbal ni se confondre avec ceux qu'elle peut sembler être 
devenue. Produire de tels objets, les vouloir, par exemple entreprendre un « sonnet nul et se réfléchissant de toutes les façons " par quelque "mirage interne des mots mêmes ", comme Mallarmé le voulut du sonnet en -yx, cela n'est certes pas sans valeur dans la recherche de poésie, car ces montages de mots eux aussi permettent d'entendre le son dans le vocable, mais pour l'essentiel ils se servent du son au lieu de s'effacer devant lui ; et en eux manque donc ce contact avec l'immédiat, avec la chose indéfaite, qui nous vaudrait un autre regard sur notre vie et le monde.

Non, la poésie est bien plutôt une réflexion sur cet être au monde que l'action ordinaire ne tente pas de complètement explorer. Et tout de suite sur cette voie elle rencontre la préoccupation religieuse, qui a trait elle aussi à la transcendance mais ne voit pas que cette dernière peut être vécue dans la moindre chose existante, ce qui la fait la chercher en un dehors introuvable de la réalité naturelle. Faut-il le dire ? À tort ou à raison la poésie est le déni de ces religions qui ne cessent de l'occulter en prétendant que leur visée englobe la sienne, et qui réussissent même à en persuader des poètes, le pouvoir souvent séculier des églises n'étant pas dans ce cas sans force de persuasion.

De ma définition du poétique découle, autrement dit, qu'il est fréquemment censuré, menacé, intimidé, empêché de se reconnaître en ce qu'il a de singulier et même d'originel. La poésie va croire qu'elle sert la cause de Dieu, voyez Dante, voyez John Donne ou Milton, elle peinera à se dégager de l'emprise de mythes qui monopolisent la transcendance, elle perdra de vue les lieux de son évidence. Et voici donc les deux grands problèmes qu'il est essentiel d'aborder si l'on veut le bien de la poésie: celui de ses travestissements et celui, en retour, de sa conscience de soi. Qui veut servir la poésie se doit d'étudier les diverses formes que cette conscience de soi a prises, dans le passé. Cette recherche aidera à éventer quelques pièges qui restent tendus même aujourd'hui.

III

Et j'en viens ainsi à Shakespeare, qui est un de ceux qui ont réfléchi à l'essence et aux périls de la poésie, sans pour autant s'être représenté clairement qu'il s'assignait cette tâche.

À Shakespeare, mais nullement pour faire part ici de pensées dont je me serais assuré, plutôt pour exposer la sorte d'étude que je crois utile de faire ; évoquant, s'il m'est permis de rêver, le livre que j'aimerais écrire sur certains aspects de cette œuvre.

Il y a des auteurs, remarquons-le d'abord, dont l'imagination crée des mondes avec lesquels leurs œuvres s'identifient. Et il y a sens alors à chercher si certains des aspects de ce vaste rêve ne sont pas la représentation symbolique de l'acte poétique, lequel aurait donc été comme pressenti dans ces textes qui n'auront pu qu'y gagner en attrait, même en pouvoir de fascination.

J'ai suggéré cette sorte d'approche dans un colloque récent sur la conscience de soi, justement, dans la poésie médiévale; et je me suis risqué à prêter ce savoir non conceptualisé - ce savoir "figural» - à quelques auteurs des romans bretons, interprétant sous ce signe l'épisode majeur de ce cycle immense. Il me semble que le "graal ", cette écuelle, cette chose on ne peut plus ordinaire à laquelle pourtant tant de foi s'attache, ce fut pour Chrétien de Troyes ou ses successeurs une façon de se représenter la transcendance que je dis simple, celle qu'est toute chose par rapport aux concepts qui veulent la dire. Après quoi la dissipation des enchantements et d'autres 
«aventures » en terre bretonne métaphorisent l'apport que cette prise de conscience, nouvelle grâce, peut être dans une vie s'ouvrant ainsi à la poésie mais aussi les maux qui découlent de sa syncope, en des moments décisifs. Pensez à l'incuriosité de Perceval au château du Roi Pêcheur quand passe devant lui, une première fois puis une autre, le cortège du Graal : le lendemain, au réveil, c'est la terre gaste, remarquable figuration de ce monde que le concept nous impose, choses réifiées, énigmes de toutes parts, fantasmes paralysant des communautés comme dans la société d'aujourd'hui la névrose le fait pour l'individu. Les romans bretons parlent de la poésie en ce qu'a celle-ci de plus spécifique, et cela fut perçu, leur succès même le prouve, comme aussi bien l'effort très cohérent, très soutenu, de l'Église pour insinuer en eux sa parole ; ce qui donna le grand Lancelot en prose.

Une conscience qui affleurait par des symboles, en l'occurrence, par un travail de l'esprit sur la signifiance de certaines choses, de certains rites. Mais ce pressentiment de la poésie peut prendre forme d'autres façons. Je pourrais évoquer en ce point ce que l'on appelle la tradition pastorale : où le poétique impensé se marque par la récurrence des situations de deuil, celles de déplorations de la perte d'êtres qui étaient beaux et moururent jeunes. " Et in Arcadia ego » c'est une façon de chiffrer « dans l'intemporel lumineux d'un rêve » l'emplacement de la lucidité poétique.

Et plus près de Shakespeare je vais faire aussi l'hypothèse que cette lucidité, cette mémoire d'un grand objet de pensée perdu, peuvent affleurer dans la forme d'une œuvre - sa forme prosodique et les retentissements de celle-ci dans le texte, style, figures autant qu'en une réflexion directe ou indirecte, consciente ou pas.

Je disais tout à l'heure que dans la réception du discours la poésie naît de la perception du son des mots, retrouvé par dessous tout emploi ordinairement sémantique ou expressif en sa réalité de son, opaque, originelle, indéfaite: une émergence, une épiphanie qui affaiblissent dans la parole l'autorité du conceptuel. Et cet instant premier, fondateur, c'est déjà permettre une réflexion qui peut prendre conscience de soi par des moyens antérieurs eux-mêmes à la signifiance explicite. D'où le sens qu'il y à interroger les événements qui scandent le devenir de l'emploi des formes à travers l'histoire. Car le son n'est perçu comme tel que si sont évacuées des mots les façons dont ceux-ci portent du sens, et ce travail du négatif, qui est difficile, ne peut que bénéficier de l'impact de la forme sur la parole. Un impact qui varie avec les époques, parce que les croyances et les pensées ne sont pas sans l'affecter grandement, avec par exemple l'effet sur les choix formels des poètes de ceux des architectes, des peintres.

Il faut questionner la forme, retrouver la pensée du poétique qui est parfois sous-jacente au travail qu'accomplit la forme sur l'écriture. Et pour cela prendre mesure de l'influence qu'a la prosodie d'une langue sur l'intuition qui guide un poète dans son emploi de la forme : cette prosodie qui est une façon d'écouter le son. Aidant à percevoir celui-ci en sa différence, peut-être en son absolu, ne risque-t-elle pas, en revanche, de le réduire à ce qui est malheureusement une fréquente lecture des poèmes : celle qui fait de la matière sonore une réserve de signifiants pour des signifiés restés à des pensées ou des rêveries que le conceptuel détermine? Pensons, avec inquiétude, aux "sanglots longs" des « violons de l'automne ». Le son est capté là au profit d'une conscience du monde autant que de soi bien conventionnelle. Ce n'est pas dans ces vers que Verlaine se montre un vrai poète. reconduit le poète en son écriture même vers à nouveau le monde comme les concepts 
l'ont produit, ce monde qui n'est qu'image. Que fait la rime, par exemple, sinon obliger chaque fin de vers à se raccorder à des mots qui avec eux apportent le dictionnaire, où tout n'est que définition par concepts? Et cela au moment où beaucoup dans l'écriture ne demande que ce retour? En effet, il ne faut pas oublier que le désir ne se dit à soi-même et ne rêve de s'accomplir qu'au moyen de représentations qui sont tout aussi conceptuelles que les formulations de la science: si bien que si le poème se fait l'expression d'un désir - et peut-il en être autrement? - l'immédiateté un instant pressentie par le poète risque bien de s'y effacer. La poésie va céder la place à ce que je dirai de l'art, du simplement artistique.

31 Mais la poésie naît d'un instant de saisissement et celui-ci peut se reproduire. Là même où l'art triomphe la poésie insiste, entendant à nouveau le son dans ce discours du désir et reprenant ainsi contact avec le savoir propre de l'être de finitude, un savoir du lieu, du temps, du hasard. Il y a conflit de deux besoins au plus secret des poètes, c'est ce conflit qui d'une œuvre d'eux à la suivante décide des voies de leur écriture. Et celle-ci, en ses expériences, est donc le plan où le poétique peut se perdre ou s'approfondir; ce qui permet de voir comment il peut être soit trahi soit servi par la forme écoutant le son. Pour retrouver dans une œuvre si la poésie y a conscience de soi, il faut comprendre ce qu'y fait le poète des virtualités de la forme.

\section{IV}

32 Cette dialectique de poésie et d'art, de forme épiphanique et de forme simplement signifiante, est particulièrement visible - et me voici entrant en pays shakespearien dans l'histoire du sonnet depuis ses origines dès avant Pétrarque puis dans le Canzoniere et son innombrable postérité.

Le sonnet est une forme conventionnelle, et cette forme est simple et forte, immédiatement perceptible, ce qui met ses règles en évidence. Que cette forme soit forte, et c'est une chance pour l'écoute poétique du son qui est au-delà, autrement dit pour la poésie, mais que ces règles soient apparentes, c'est une incitation à valoriser la forme comme telle, avec ses capacités d'organisation de la perception et de la pensée, ce qui va retenir au sein du monde-image qui conditionne la société de l'époque. Le sonnet est donc un parfait terrain pour la concurrence du poétique et de l'artistique, mais aussi et de ce fait même pour la conscience de soi de la poésie.

Or, précisément, il a connu à travers l'Europe pendant deux ou trois siècles de mutations extraordinaires la vogue que nous savons. N'est-ce pas le signe que s'y est produit quelque chose d'important, et précisément à ce plan fondamental ?

35 Je n'en doute pas, et remarquerai que du point de vue de la poésie, qui est la substitution de la transcendance du simple à celles dont rêve la religion, Pétrarque, le premier grand du sonnet, est tout à fait digne d'attention. Homme de religion tant qu'on veut, mais aussi celui qui fixe ses yeux sur le Ventoux, une cime où personne ne s'intéresse à grimper, et prend contact avec lui en en faisant l'ascension. Qu'est-ce que le Ventoux, en l'occurrence, sinon la chose naturelle en ce qu'elle a d'impénétré, de transcendant par rapport à la pensée ? Alors que Pétrarque le bon chrétien aurait dû n'avoir de regard que pour les sphères concentriques $d u$ cosmos que Dieu a créé, d'oreille que pour leur musique supraterrestre, il ne peut s'empêcher de penser à cette montagne qui n'a pourtant nulle place dans la réalité symbolique et immatérielle qui lui importe, ce qui 
prouve qu'il est sensible à ce que le Ventoux ne doit qu'à son propre fait. Le même appel que chez Chrétien de Troyes ou d'autres faisait entendre le graal, chose quelconque. Et de la part de Pétrarque, indéniablement, une disposition à la poésie.

Ceci étant je n'oublie pas que parvenu au sommet du Mont Ventoux et découvrant de làhaut le monde comme il existe, une étendue où le hasard prédomine visiblement sur toute structure que Dieu aurait pu vouloir, Pétrarque s'inquiète, se fait, littéralement, taper sur les doigts par saint Augustin dont il avait pris avec lui un livre; et rentre précipitamment dans l'orthodoxie. Mais ce retour à ses habitudes n'est pas sans perplexité, d'où la relation qu'il écrit de son aventure. Et l'important, c'est que cette même dualité de curiosité du monde et de foi se découvre dans ses sonnets, comme s'il les avait écrits pour continuer à la vivre.

Que constate-t-on, dans le Canzoniere ? D'abord la relation ambiguë que j'ai évoquée tout à l'heure, entre un désir personnel, sexuel, celui qui se porte sur Laure, une jeune femme, et le discours du groupe, qui prône de tout autres valeurs et privilégie d'autres figures. La Laure des sonnets est apparemment en règle avec l'orthodoxie catholique qui valorise l'image de la Vierge, toute chasteté, toute réserve, et c'est au point qu'on peut lire ces poèmes comme on regarde un retable de Simone Martini : leur figure centrale est aussi entrônée et environnée de figures symboliques qu'une Madone de ce grand peintre. Mais quelque chose d'autre se marque, au moins fugitivement. Voici, par exemple, c'est le sonnet 34, que l'amant de Laure, troublé, demande à Apollon - le dieu de la poésie, remarquons-le - de venir avec lui contempler la dame de ses pensées assise dans l'herbe, au soleil mais se protégeant de l'ardeur du ciel par ses deux bras réunis, belle imagination de peintre plutôt cette fois impressionniste. Qu'est-ce que cette proposition signifie ? Que Laure est vue maintenant par Pétrarque comme une personne réelle, avec sa pleine apparence, au-delà aussi bien des réductions que le désir accomplit que de celles qu'impose le moralisme chrétien. Paraît dans un poème, qui en devient de la poésie, une personne en son évidence irréductible autant qu'insondable, la même transcendance sans mythes que Pétrarque avait reconnue mais aussitôt fuie sur le Ventoux.

La transcendance inhérente à la réalité ordinaire a pris la place de celles que la religion ou le rêve imaginent au cœur de leur transfiguration de ce qui est. Une transcendance réelle se laisse à tout le moins entrevoir là où il n'y avait dans la culture de cette époque que des transcendances fictives, ce qui fait que dans ce sonnet 34 un désir lui-même tout simple se mue aisément, naturellement, en un bien plus véridique et profond amour. Tel est le travail de la poésie.

Un travail qui tout autant peut échouer et laisser paraître son contraire, c'est-à-dire la réduction d'un objet d'amour - d'un être reconnu en cette transcendance que je dis simple - à seulement une figure idéale, du fait d'une écriture reprise par les réseaux d'une image. Cette figure centrale est éprouvée par le poète comme encore une transcendance, mais c'est la sorte de transcendance rêvée que met en perspective un intelligible, c'est l'Idée comme s'en réclament les platoniciens de la Renaissance.

\section{V}

Il en est ainsi, ou plutôt il semble au premier regard qu'il en soit ainsi chez Ronsard dans l'admirable sonnet 49 du second livre pour Hélène, celui qui commence par «Le soir qu'Amour vous fist en la salle descendre ». L'époque de ce poème, c'est justement celle où 
une philosophie héritée du platonisme a accoutumé à penser en termes d'intelligible, à chercher à rejoindre l'être par la beauté de la forme, et le sonnet 49 peut ne paraître rien d'autre que la mise en évidence de cette transmutation, cette Hélène pourtant réelle semblant valoir par sa connivence avec les formes d'une danse à laquelle elle se joint en vraie souveraine, ce ballet dont les mouvements successifs, cercles, triangles, sont des figures géométriques à la façon des sphères célestes. « Divin » est le ballet. " Transformé » le corps de la femme. On peut bien croire que Ronsard a cédé à la force de conviction qui est en prosodie dans les formes fixes et consenti de ce fait à ne reconnaître réel que ce qui participe d'un intelligible, d'une harmonie entre formes.

Et ainsi aurait-il trahi, en cette Hélène au dessus du monde, cette affirmation de la finitude que je dis le vœu de la poésie. Mais peut-être Ronsard a-t-il surtout voulu dans ces vers expliciter l'autorité de la forme dans l'écriture, ce qui serait déjà un acte de grande lucidité. Et même je pense qu'il y a entrepris bien plus. Il n'y a pas qu'une pensée de la poésie dans le sonnet qui dit qu'Amour fait descendre Hélène dans la salle, il y a, c'est évident, c'est bouleversant, la poésie même. Et il est facile de voir pourquoi.

Que dit Ronsard, en effet? Que les formes, que la musique des formes, exercent dans cette salle mystérieuse leur grand prestige, c'est vrai, mais aussi qu'Hélène dans ce ballet en est seulement une part ; et que si elle participe en lui et par lui à ce qu'il a de "divin ", elle n'y est nullement perçue comme l'Idée même, inaccessible au point de fuite des formes, mais comme, bien que menant le jeu, un être de notre monde, dont le bonheur à la danse, bonheur anxieux, donne relief à ce que son corps a d'humain encore et à jamais, de mortel.

Et ce ballet, d'ailleurs ? Est-il seulement « en triangle » ou « rond », signe d'un divin qu'il aurait rejoint - « car la perfection consiste en chose ronde », a écrit Ronsard, autre part -, non, il se reprend, il se rompt, il se refait, il contre-imite «le cours du fleuve de Méandre ", lequel, on le sait, est le hasard même : il veut la forme pure, c'est sûr, comme "l'escadron de la Gruë évitant la froidure ", mais dans ses figures il n'en préserve pas moins les aspects de notre réalité sublunaire, on dirait même qu'il veut en maintenir l'être propre jusqu'au plus haut de sa pensée de la forme. Qu'est-ce que ce ballet qui dit à la fois la forme et le non-formel?

Eh bien, c'est une allégorie, une image en abîme, du sonnet lui-même, mais de celui-ci en tant cette fois que poésie, vraie poésie : c'est-à-dire la persistance du savoir de la finitude là même où la prégnance d'une structure formelle tend à imposer son intemporel. Est-ce du fait de cette ambiguïté à la fois dite et vaincue que le ballet est « divin », est-ce à cause d'elle, à la fois la contrainte et sa transgression, qu'il transporte Hélène « sur le haut de la terre» et que le corps de la jeune femme - son corps, non son esprit - est «divine nature » et " pour ce soir ", c'est-à-dire pour simplement une heure mais celle-ci hors du temps? En tout cas ce grand poète donne bien à méditer cette dialectique du poétique et, qui plus est, il le fait non par d'abstraites formulations mais en faisant paraître ce qui est l'apport de la poésie, précisément: une présence charnelle, un corps en sa vie la plus immédiate, une musique mais reçue ici et maintenant et par un corps aussi bien qu'un intellect.

45 Le sonnet 49 dit le péril de la poésie mais en ce péril où elle pourrait se renoncer, se faire seulement un rêve né de la forme, il réaffirme la grande intuition certes toujours entravée qui en est l'essence. Ce poème est un bel exemple de conscience de soi de la poésie, de conscience active. Et c'est pourquoi je m'y suis attardé : il montre que le sonnet 
est éminemment capable d'une réflexion sur les pouvoirs de la forme mais aussi, de Pétrarque à Ronsard, de moments où il se défait de sa contrainte.

\section{VI}

de vue de la conscience de soi de la poésie. D'autant que Shakespeare conçoit cette scène, où sont vilipendés les vers qu'Hamlet avait dédiés à la «beautified» Ophélie, presque aussitôt après les quelques années où il écrivit lui-même plus de cent cinquante sonnets, après quoi, assez brusquement à mon sens, il s'en détourna pour se concentrer à nouveau sur son travail au théâtre.

\section{VII}

L'heure passant, je m'en tiendrai maintenant à quelques remarques sur les sonnets de Shakespeare. À juste ce qu'il faut pour inciter les lecteurs à penser que ceux-ci sont du plus grand intérêt du point de vue qui est mien, réfléchir sur ce qu'un poète peut avoir pensé de sa propre pratique de l'écriture.

Actes des congrès de la Société française Shakespeare, 24 | 2010 
51 Une première remarque portera sur le caractère tout de même assez singulier de leur occurrence, au milieu des années 1590, au plus fort de la grande mode du sonnet en Angleterre. Voici un auteur qui s'est également illustré par son théâtre et sa poésie, l'un largement connu du public et riche déjà de grandes œuvres, l'autre, la poésie, appréciée par des amateurs moins nombreux mais qui l'estiment, n'en doutons pas : car les poèmes que Shakespeare a très récemment écrits, Venus and Adonis et The Rape of Lucrece, ne sont nullement les œuvres d'un habitué de la scène qui penserait à la poésie les jours de relâche, on y trouve toutes les qualités d'un professionnel rompu à sa pratique. Or, c'est précisément alors, c'est en ce point d'équilibre entre deux tentations, deux maîtrises, que Shakespeare en vient aux sonnets et, peut-on penser, pour un temps assez bref, après quoi il n'écrira plus de poèmes - sauf une fois, en une occasion - et se consacrera entièrement au théâtre, au seuil alors des chefs d'œuvre de celui-ci. Les sonnets ne sont donc pas un de ses ouvrages parmi les autres mais ce qui va mettre un terme à une des branches d'une activité de ce côté-là aussi on ne peut plus prometteuse, autant que susceptible, socialement, de valoir considération à un auteur.

Clairement, quand il cesse d'écrire ses sonnets, Shakespeare accomplit un choix, et puisque ce poète est un grand esprit et au faîte de ses moyens il y a tout lieu d'estimer qu'il n'a pas fait ce choix par hasard, pour des raisons contingentes, mais en y engageant une réflexion, laquelle, sommes-nous en droit de penser, va être perceptible dans ses écrits du moment. Perceptible et interprétable, ce qui pourra éclairer la question de la poésie comme Shakespeare l'entend, en particulier dans son rapport au théâtre.

quelques autres remarques, des préliminaires encore, sur cette fois le texte de ces sonnets. La première, c'est que ceux-ci n'ont rien d'autobiographique, sauf peut-être pour des détails qui n'en affectent ni la visée ni le sens. J'ai lu, sous une plume critique, que les sonnets sont le seul ouvrage de Shakespeare où l'on entende sa voix : mais ce qui est vrai c'est tout le contraire. On entend partout Shakespeare, dans son théâtre mais je ne sens pas que dans ses poèmes il exprime ou transpose quoi que ce soit de sa vie, ou d'un sentiment qui lui serait personnel, sauf peut-être dans deux ou trois des sonnets, dont le 129 , sur la luxure : «Th'expense of Spirit in a waste of Shame ».

Partout dans son théâtre, Shakespeare, partout avec sa voix et toute sa voix ? Oui, dans Hamlet, par exemple. Tout au long des hésitations, des reprises, des sautes d'humeur, des décisions imprévues du prince de Danemark, qu'y a-t-il d'autre que l'émergence d'une intériorité encore si proche de sa source qu'elle est en mal de mots pour se dire? D'évidence cette parole est surchargée de sentiments incompris, d'associations inconscientes, elle trébuche sous ces entraves, or, l'inconscient, cela ne s'imite pas, et cette façon de parler sur scène, si neuve alors, ne peut donc naître, serait-ce au plus vif d'une action, que de celui qui écrit la pièce : qui l'écrit à la diable, peut-on penser, ne sachant pas à l'instant d'une réplique de quoi la suivante sera faite. Je ne doute pas qu' Hamlet ait été produit très vite, par quelqu'un qui débordait de soi et se fiait à son intuition.

Tandis que les sonnets! Même ceux qui semblent le plus secoués par une expérience vraiment vécue, ainsi celui déjà mentionné sur la luxure, même ceux-là n'évoquent ce qui les hante qu'en termes très généraux, très impersonnels, avec une pléthore de lieux communs qui sont souvent de la banalité la plus éhontée, du genre : «Qui peut résister à une femme? » ou "mon amour, une fièvre ». Et ces stéréotypes les structurent comme des pensées que l'on tiendrait pour fondamentales, jamais à soumettre à critique. Dans le théâtre de Shakespeare, les comédies ou même les tragédies, des affirmations de cette 
nature paraissent bien, mais alors comme des traits caractéristiques d'un personnage, vus comme tels - ainsi les lieux communs proférés par Polonius, dont Hamlet se raille, ou ceux plus timides de Celia au début d'As You Like It, immédiatement relevés par la parole ironique de Rosalinde. Prédomine en ces cas un souci d'analyse psychologique, à visée souvent très profonde.

Mais les lieux communs des sonnets se situent, eux, en deçà de toute situation où ils seraient observés, critiqués, voire moqués, on ne peut que les estimer tout à fait assumés par celui qui tient ce discours où ils se succèdent, son fait d'ailleurs et jamais celui de personne d'autre. Ils ne sont pas l'objet d'une réflexion mais son instrument, les composantes d'un être au monde dont ils font entrevoir la capacité d'intellection, d'émotion.

Et celle-ci, vue ainsi, ne semble guère admirable. Car que se passe-t-il quand des lieux communs de cette sorte figée régentent une parole, comme s'ils étaient les atomes d'un univers dont celle-ci ne ferait qu'enregistrer la structure? Ils établissent un niveau de pensée au plan duquel les êtres comme ils sont ou les choses ne peuvent laisser paraître ce qu'ils ont en surcroît de ces perceptions schématiques; et c'est le souvenir de cette profondeur, avec ses imprévus, et sa vérité propre, celle de l'existence particulière, à la fois finie et infinie, qui s'efface de la conscience, laquelle en est appauvrie. Elle va se méfier de cet en plus qu'elle ne peut pas totalement méconnaître, elle en aura même peur, faisant de lui du négatif, du nocif : comme le montre dans les sonnets la «dark lady ", cette image si triste de la femme et de ses supposées façons d'être. La "dark lady ", la femme moins brune que noire, d'un noir d'essence métaphysique, n'est que sexualité menaçante, ses sollicitations vont faire que l'homme sombre dans la luxure puis dans la honte de soi : on voit sur cet exemple que s'en tenir à des lieux communs, ne pas les mettre en question, c'est se vouer à croire à l'existence du mal.

Et c'est se vouer aussi à concevoir un bien de même nature irréelle, à preuve cette fois le beau et vertueux jeune homme que le «je » des sonnets ne cesse de célébrer, alors que dans ce champ d'abstractions il n'est qu'une image encore, sans enracinement dans de l'existence, ce que reconnaît son dévot puisque celui-ci s'inquiète de ne pas le voir procréer, c'est-à-dire faire acte d'incarnation. Il s'en inquiète, il ne devrait pas pour autant s'en étonner. Image, et composée de traits qui ne sont beauté et vertu que par simplifications aussi grandes, le beau jeune homme ne peut que vivre enclos en son essence d'image, et ce que son ami lui reproche, c'est moins le narcissisme ordinaire que la fatalité de son irréalité.

Cet aspect des sonnets de Shakespeare les rend bien différents, remarquons-le maintenant, de ceux de Pétrarque ou de ses disciples, dont le dernier en date, Sidney. Différents? Oui, parce que chez Pétrarque ou Ronsard ou Sidney déjà les épithètes, les attributs ont beau être conventionnels, schématiques, ils ont beau ne donner forme qu'à des figures peu capables d'incarnation, ils n'en cherchent pas moins à rendre compte d'un être qui existe dans la pensée de ces poètes à plus haut niveau que tous les autres, avec de ce fait la tâche de signifier une pleine réalité, quelque chose même de plus intense que l'exister dans ce monde. Laure n'est-elle qu'une image, oui, mais Pétrarque suggère que des rayons en émanent, comme d'une Vierge dans un retable. Et suit de cela que, tels les anges autour de cette Vierge, les adjectifs des sonnets de la tradition pétrarquiste ont quelque chose, dirai-je, d'exclamatif, ils restent les prisonniers de leur abstraction mais ils tentent de soulever celle-ci vers bien plus, et même bien plus que du réel ordinaire. Serait-ce en rêve, ils voudraient accéder à une présence. 

voie de Laure ou des autres femmes de même rôle, ils cherchent cette transcendance au centre, tout de même, de la réalité ordinaire, cette dernière a beau être abordée par ces lieux communs, de façon abstraite, elle n'en a pas moins pour eux un arrière-fond, un rayon vient quelquefois s'y poser sur simplement une prairie, une fleur, et c'est moins pour s'y perdre qu'y ressourcer son mystère. Ce n'était pas un hasard si Pétrarque avait prié Apollon, le dieu, de venir avec lui contempler un peu de ce monde. Le pressentiment du surcroît de ce qui est sur les figures qui l'emprisonnent hante les sonnets depuis Pétrarque, c'est en cela qu'ils peuvent être vraie poésie.

61 Et pourtant, rien ou bien peu de cette dialectique de présence et de figure dans ceux qu'a écrits Shakespeare. Par rapport à la dame brune, le beau jeune homme, aussi blond soit-il, n'est pas comme Laure une transcendance, il peut en subir des atteintes, perdre de son fait sa vertu : la femme qui est mauvaise et l'homme qui est le bien sont à même niveau dans un système d'oppositions, de tensions qui n'est pas une remontée vers plus que le monde mais une simple structure conceptuelle, avec son risque fondamental, lequel est de perdre de vue la réalité particulière, infinie autant que finie, et cette unité de tout qui affleure pourtant dans la moindre chose. Dans cette algèbre de relations d'emblée décidées, pas de communication avec le dehors. Et le débat des trois personnages l'homme, la femme, l'auteur qui est leur ami - n'aura donc lieu qu'en un champ de psychologie ordinaire mais aussi de préjugés et de distorsions. La conséquence étant qu'on peut craindre qu'il n'y ait là qu'une possibilité de mauvais théâtre; et que les beautés de cette écriture aient à être surtout un fait de langue, de mots employés avec d'autant plus d'art qu'on ne leur demandera guère de s'inquiéter de la vérité. Et, de fait, il y a beaucoup d'habile rhétorique dans le discours des sonnets, et la musique de leurs vers est souvent exquise. Des divertissements sur la scène, qui valent plus que le drame que l'on y joue.

Mais en venir à penser cela, quand il s'agit de Shakespeare, c'est encore avoir à se poser des questions, et de bien grandes.

De l'art et non de la poésie, ce n'est évidemment pas ce qui caractérise Shakespeare. Et parler de «mauvais théâtre », comment en prendre le risque quand il s'agit de lui, et dans ces années où il a écrit les sonnets. C'est en effet le moment où il approfondit brusquement sa recherche de dramaturge avec Romeo and Juliet et A Midsummer Night's Dream, après ce seront The Merchant of Venice et deux ou trois ans à peine plus tard As You Like It puis Julius Caesar et Hamlet dans la nouvelle salle du Globe. Au moment où il cesse d'écrire ses poèmes, Shakespeare rejoint les sommets de sa création et en comédie et en tragédie. Il importe donc, réfléchissant aux sonnets, d'en déplacer le problème. Il convient de se demander pourquoi celui qui devient si grand poète au théâtre se préfère, dans ces sonnets, simple artiste. Quelle motivation, quelle préoccupation l'ont poussé, quelle expérience a-t-il faite, qu'il faut chercher à comprendre? La réponse à cette question ne pouvant qu'avoir trait, sous cette vêture, des poèmes, au plus essentiel de la poésie.

\section{VIII}

Je vais chercher à comprendre cet apparent désaccord du sonneteer et de l'auteur dramatique.

Actes des congrès de la Société française Shakespeare, 24 | 2010 
Et pour ce faire j'en reviens à ce que j'ai dit plus haut de la poésie, donnée pour la transgression dans les mots de la lecture du monde que fait l'instrument conceptuel, lequel ne sait rien de la finitude - mais aussi comprise comme l'échec, quasi obligé, de cet acte de transgression. Cet échec, le poème, c'est du conceptuel à nouveau, de l'abstraction comme dans le discours d'avant, mais avec une liberté que ce dernier n'avait pas et une capacité réflexive qui permet au poète de comprendre ce qui l'entrave et de partager avec son lecteur cette conscience de soi. Deux moments successifs, en somme, bien qu'ils ne cessent de se rejoindre dans une seule intuition. De l'art se substituant à la poésie première mais pour s'offrir à critique et permettre au poète de se mieux connaître, de visiter ses limites, d'appeler une société à partager son projet.

Je définis ainsi la poésie et ce n'est pas, on le voit, sans retrouver certains des caractères que j'ai cru pouvoir discerner dans les sonnets de Shakespeare. Y reconnaître l'importance déterminante d'un réseau de stéréotypes privant les protagonistes du drame qui s'y joue d'être autre chose que des abstractions eux-mêmes, n'est-ce pas, en effet, les rejoindre au niveau en eux de ce que j'appelle le "poème » dans le travail de la poésie ? Sauf que les vrais poètes ne veulent pas rester prisonniers à ce plan, se retournent de celui-ci vers leur intuition première, demandent à Apollon d'aller avec eux vers Laure assise au demi soleil de l'orée d'un bois, tandis que les lieux communs du sonnet shakespearien semblent faire preuve d'une satisfaction à eux-mêmes qui éteint dans ces vers le souvenir de la poésie.

Embarrassante constatation! Dois-je en conclure, avec cette idée de la poésie, qu'en ses sonnets en tout cas Shakespeare ne sait pas ce qu'est celle-ci, ce qu'elle demande?

Non, plutôt m'aviser que cette complaisance aux clichés, au soleil qui est d'or, à la femme qui est mauvaise, etc., est tout de même bien grande, dans un texte et chez un auteur où pourtant il est clair que sensibilité et intelligence foisonnent. Et commencer à soupçonner qu'il s'agit moins là d'une adhésion simple de Shakespeare à des points de vue schématiques et réducteurs que son désir de donner relief à ce qui se joue dans la création poétique entre intuition première et poème : son travail sur ces vers, ou déjà dans Venus and Adonis ou dans The Rape of Lucrece lui ayant montré que le mot un moment dégagé du discours usuel du concept redevient vite, dans le poème, concept encore. À nouveau un système de représentations au lieu de la pleine présence, à nouveau cette abstraction et ces préjugés là où conscience était prise, ou presque, de l'être en sa finitude, de l'Autre en sa vérité, du Je en sa liberté : et cela, même avec grande richesse d'art, de musique, de rhétorique, c'est bien du stéréotypé, n'est-ce pas ? Et de quoi s'inquiéter, si l'on est poète et lucide, de quoi se sentir requis de regarder de plus près ce qui se passe dans ce qu'on fait. D'un mot: on regardera à la loupe cette écriture qui va son cours, et ce seront des grossissements qui la montreront de même nature, hélas, que les lieux communs et clichés qui nous ont étonné chez Shakespeare; et même nous donneront à penser que ces derniers ne sont qu'une façon de signifier l'effacement de la poésie, cette transgression, au sein d'un discours de simple visée artistique. L'étouffement d'une parole qui était libre par les structures rigides, les abstractions, d'une langue fermée sur soi.

Mon hypothèse, vous le voyez, et ce serait découvrir un bien bel exemple de cette conscience de soi du poétique que j'ai appelée figurale: Shakespeare dans ses sonnets a mis en évidence le réseau de représentations conceptualisées qui, au moment "poème " du poétique, remplace de ses signifiés la perception pleine un instant vécue. La pauvreté manifeste des lieux communs où ces écrits semblent se complaire, ce serait pour coder, 
pour désigner cette chute. Shakespeare aurait grossi dans le discours du poème, pour le montrer, ce par quoi celui-ci cesse d'être poésie.

Mais comment a-t-il pu se résigner, même inconsciemment, à cela, va-t-on me dire ? Car un poète comme lui aurait dû être incapable de résister, écrivant son poème, au désir de le reconduire à la poésie: cherchant alors à décomposer la structure conceptuelle recommencée au lieu de la dilater pour la contempler. Ce travail de la poésie sur le poème, n'est-ce pas quelque chose d'irrésistible si l'on n'est pas qu'un pauvre rimeur? C'est lui que nous avons vu à l'œuvre chez Pétrarque ou Ronsard, et nous le constaterions tout autant dans tous les beaux sonnets de l'histoire et non seulement dans ceux-ci mais dans tous les emplois de la forme fixe, lesquels ont même fini par en être totalement disloqués dans notre moderne vers libre. Comment Shakespeare a-t-il pu ne pas être poète, en somme?

\section{IX}

Comment, pourquoi? Mais prenons conscience maintenant de deux données fondamentales de sa condition de poète dans ces dernières années $\mathrm{du} \mathrm{XVI}{ }^{\mathrm{e}}$ siècle.

D'une part, dans la société littéraire, c'est cette vogue des sonnets, avec ce qu'elle apporte de médiocrité, d'évidente superficialité, dans des textes où pourtant le sonnettiste éhonté ne se prive pas des exclamations qui se prétendent le signe d'une expérience profonde: de toutes parts un lyrisme factice, bien de quoi irriter ceux qui savent ce qu'est la poésie authentique. Bien de quoi les pousser à la dénonciation de ces contrefaçons du véritable lyrisme.

Et d'autre part chez Shakespeare une pratique de la parole scénique déjà ancienne et assurément chevillée en lui de la façon la plus forte, puisqu'elle a été son expression première, toute spontanée, et a donné lieu d'emblée à des œuvres aussi assurées d'ellesmêmes que Love's Labour's Lost ou Richard III. Cette parole, qui court à sa vérité à travers des situations observées avec sympathie dans les lieux et moments les plus variés de l'existence contemporaine, est poésie, elle aussi, par sa transgression des points de vue convenus, fréquente brusque trouée dans la masse des discours de tous et de toutes réseaux de lieux communs s'il en est - vers un au-delà de pleine immédiateté dans des instants d'existence. Et elle est poésie d'une façon que le dramaturge peut cette fois sentir libre d'aller hardiment de l'avant: le vers que Shakespeare emploie - ce pentamètre sans trop de rimes, aux mots accentués comme dans la parole ordinaire - n'ayant pas à briser son flux sous la contrainte des formes fixes. Cette poésie de la scène, qui culmine dans de vastes poussées verbales - ainsi les premiers vers de 1 Henry IV, « So shaken as we are, so wan with care... » - a une ampleur d'horizon dont ne bénéficient certes pas même un Spenser ou un Sidney dans leurs livres; et elle assure un arrière-plan de pratique, et de confiance, dont il ne faut pas sous-estimer l'importance quand Shakespeare a l'obligation de se renfermer dans quatorze vers.

Ces deux données, et alors cette conséquence, bien naturelle: Shakespeare jette sur le sonnet qu'il écrit le regard d'un homme de théâtre qui, poète instinctivement, s'inquiète de voir les mots se refermer sous sa plume, se flétrir, mais sait aussi que ce n'est pas dans l'écriture sur une page qu'il a son plus grand pouvoir de leur rendre vie. Et simultanément il constate que cette contrainte des formes fixes a des effets sur sa conscience de soi qui lui expliquent ce qui a lieu dans la société, semblablement soumise à 
des impératifs dans la langue, à des formes qui la contraignent. Le sonnet est un miroir où les rapports sociaux sont visibles en ce qu'ils ont de plus radical. On peut y déchiffrer le drame qui dresse les uns contre les autres des êtres enfermés chacun dans les abstractions de la langue, voués ainsi à se méconnaître. Et prendre mesure de ce drame, le mettre en scène, c'est bien ce que fait Shakespeare, comprenons-le maintenant.

Car ce n'est pas seulement un réseau de lieux communs qui caractérise ses sonnets, c'est des uns aux autres le déploiement d'une action comme on en voit au théâtre, bien qu'à plus haut niveau dans l'appréhension des conduites que celui où semblait se cantonner dans les œuvres premières de Shakespeare son idée d'un ivrogne anglais ou d'un marchand vénitien. Dans cette économie de figures que je disais tout à l'heure toutes situées sur un même plan, celui des concepts, sans transcendance possible pour l'une aux dépens des autres, nous voyons l'homme d'abord, un beau jeune homme, se refermer sur une image de soi, idéale, qui n'est qu'une illusion et le prive, en miroir, de sa vie même ; puis nous voyons en face de lui la femme, celle-ci empêchée par cette même idéalité qui pénètre tout d'être perceptible pour ce qu'elle est - autrement dit ni bonne, nécessairement, ni mauvaise - et en est donc réduite, elle aussi, c'est le second meurtre sur cette scène, à n'être plus qu'une image. Mais, cette fois, reste d'elle par en dessous cette image un peu de présence inquiétante, crainte, car il lui faut assumer l'irrégularité de la vie, l'obscur de la finitude, tout ce dont le rêve du beau jeune homme prétend qu'il peut se défaire. Elle sera le mal en face du bien. Et c'est comme une transcendance qui tend à se reformer dans cette structure conceptuelle, celle de ce bien sur ce mal, sauf que ce n'est plus comme chez Pétrarque un essai de transmutation des données sensibles, avec en esprit du supraterrestre, la sorte de transfiguration que les retables siennois suggèrent. Tout reste dans l'espace social ; dans des pensées, des opinions, des conduites.

\section{$X$}

76 Et combien est-il important que sur cette scène de simple métaphysique sociale la figure idéalisée soit un homme, et non la jeune femme qu'aurait pu imposer à quelque nouveau poète le lyrisme traditionnel ? Pourquoi un homme, comme objet de l'adoration éperdue bien que non mystique d'un autre homme, celui qui dit « je » dans le texte? Bien erroné serait-il d'expliquer cette innovation de Shakespeare par ses tendances homosexuelles, réelles ou supposées, car l'homme au lieu de la femme, c'est ici une réflexion sur la société comme elle est. La société ? Un espace où la femme est déjà perçue en image, ce qui permet de la méconnaître. Car cette image est produite par des valeurs masculines, si bien qu'à s'enthousiasmer d'une femme qui incarnerait, si c'est le mot, ces vertus, on ne fait que retrouver ces valeurs, aux dépens d'une réalité vite soupçonnée ou même diabolisée. Des louanges de cette sorte ne font évidemment que rendre plus difficile, chez par exemple Pétrarque, le travail de la poésie, c'est une des raisons de leur affinité comme originelle avec le triste génie des formes fixes.

77 Et déplacer vers un homme le pôle de l'adoration et voir à partir de lui les effets de l'idéalité sur la vie, c'est dégarnir la figure féminine de ce statut ambigu, valorisation mais aussi mépris, faire apparaître au plein jour la femme noire cachée dans la rêverie pseudo poétique, laisser monter avec elle le flot des opinions reçues, des lieux communs, des stéréotypes, en bref c'est se retrouver dans le lieu réel de l'existence sociale, où les femmes sont toutes des «dark ladies» en puissance dans le psychisme d'hommes conditionnés par ces lieux communs et épris de leur propre image, ce qui explique le 
meurtre de Desdémone ou le désir de changer de sexe qu'éprouve lady Macbeth ou le besoin qu'exprimera Cléopâtre d'être reconnue en sa dignité, sa "noblesse ». De ce point de vue les sonnets, cette célébration de ce qui n'est pas, le beau jeune homme, sont une analyse lucide de ce qui est, où s'annoncent de façon en fait très précise les grandes tragédies shakespeariennes.

Quant au «poète " qui y paraît comme à la fois leur auteur et un des acteurs sur leur scène, il complète bien ce tableau d'une société aliénée puisque, idolâtre du beau jeune homme et donc contempteur des femmes, il laisse voir qu'il ne gagne pas à s'empiéger dans ces vues, à preuve son étonnement devant ses propres inconséquences, ses enorgueillissements qu'il sait dérisoires, et surtout - cela pour nous, les lecteurs - sa soumission non critique à une parole sans ouverture sur le réel : puisque c'est bien de lui que jaillissent sans fin les jugements stéréotypés, les fleurs de rhétorique d'emblée flétries, tous ces aspects de la domination d'une langue figée sur la parole dont Shakespeare a fait dans cette œuvre son poste d'observation. Ce supposé poète, métaphore lui-même, celle des auteurs de sonnets en ce temps de leur grande vogue, vat-il pouvoir se désenchaîner, se clarifier? Que vont devenir ses protagonistes - mais qui sont aussi bien ses créations - dans un drame qui est celui de la société mais n'en est pas moins, plus en profondeur, celui du rapport d'un poète aux mots, celui même de l'écriture? Tel est le "suspense » de ce drame, un suspense qui ne se résout pas, tout ayant lieu ici dans l'espace d'une langue qui impose son abstraction.

Que fait Shakespeare dans ses sonnets? Il visite les soubassements des pièces qu'il a écrites ou va écrire, celles-ci cette fois de vraies comédies ou tragédies parce qu'au niveau d'une écriture plus libre il a pu et surtout pourra rencontrer le temps comme il est dans la vie réelle, avec son enseignement de finitude dans ces hasards qui en sont l'essence. Shakespeare étudie, dans ces poèmes, il découvre des vérités. Et ce grand poète peut donc se dispenser de chercher dans leur écriture des bribes de perception poétique, comme le firent Pétrarque ou d'autres dans le vase des formes fixes, car il est tout à cette analyse des composantes premières de la conscience de soi, celles mêmes qui vont lui permettre d'approfondir demain ses créations théâtrales: de pénétrer, au-delà d'un prince Hal ou d'un Falstaff observés surtout par le dehors des conduites, les méandres de la pensée, même de l'inconscient, d'un Brutus, bientôt d'un Hamlet. Shakespeare est entré dans l'espace du sonnet non pour sauver celui-ci, pour le rendre à la poésie, mais pour méditer la structure de l'être au monde social, victime de valeurs et de représentations dogmatisées, sclérosées.

80 Et c'est aussi, remarquons-le pour finir - ou pour commencer, si j'avais le temps -, une réflexion qu'il fait sur sa propre vie et son projet d'écrivain, au moment le plus important de sa carrière.

81 Cette réflexion est presque explicite dans les pièces qu'il a écrites à l'époque des sonnets et juste après, c'est ce que j'ai cru pouvoir commencer de montrer dans une introduction, naguère, à As You Like It. Aujourd'hui, je ne puis que mentionner ce surcroît de lucidité que Shakespeare a gagné de son incursion dans les formes fixes, n'en évoquant, rapidement, qu'un exemple : ce fait que Roméo, le chantre d'une transfigurée Rosaline, le poète lyrique, l'auteur, vraisemblablement, de sonnets, c'est aussi celui qui sous le balcon en cela funeste fait de Juliette une image, une Laure ou une Stella, après quoi toute une nuit entre le rossignol trop aimé et l'alouette maudite ne réussira pas à défaire ce besoin qu'il a de produire de l'idéal, d'où sa fuite, qui en dit long, et toute la suite. Cet amour de Roméo, c'est de la passion, comme on dit, mais la passion est un rêve qui détruit l'être de 
ce qu'il aime, et de cette passion, là est l'apport de vérité de la pièce, Juliette, en effet, va mourir, et même de façon non plus seulement métaphysique mais tristement ordinaire. Le balcon, le sonnet, mènent droit à la tombe, sous les yeux de Mercutio par lesquels Shakespeare regarde. Et ce n'est peut-être pas un hasard si cette tragédie dont le pseudo lyrisme est la cause débute par un sonnet, inusuellement.

Toutefois ce n'est pas seulement la problématique des comédies et des tragédies shakespeariennes que l'on découvre affectée par l'expérimentation des sonnets, c'est leur qualité poétique. La visite des stéréotypes a révélé à Shakespeare, par contraste, le fait dans le sonnettiste de la personne réelle, et ç'a été pour lui des questions sur soi, de l'inquiétude, mais aussi une exaltation de sorte nouvelle, un sentiment de sa responsabilité devant soi et devant les autres. Et ce «Je » plus profond perce désormais, qu'il le sache ou non, dans les personnages qu'il crée, leur conférant une vie, les enracinant de façon directe, pour la première fois sur la scène anglaise, dans le riche terreau obscur d'une subjectivité au travail. J'ai déjà souligné, avec bien d'autres sans doute, qu'Hamlet est directement celui qui écrit Hamlet. Il parle comme le fait une personne réelle, dans l'ignorance de soi, et s'éclaire d'ailleurs de quelques exclamations qui dans les sonnets étaient pour une fois, on n'en peut guère douter, un pleinement vécu bousculant de son émotion les conventions rhétoriques.

Je pense à l'angoisse d'Hamlet devant les occasions manquées, le temps perdu, à sa honte de soi - «0, what a rogue and peasant slave am I!»-, je pense alors à «the expense of spirit in a waste of shame ", dans le célèbre sonnet sur la luxure, et c'est me demander si on ne gagnerait pas à poser plus qu'on ne le fait d'ordinaire, me semble-t-il, la question de la sexualité de l'étrange prince, peu dite dans la pièce mais tout de même bien clairement évoquée par son agitation, vestimentaire, gestuelle, devant Ophélie effrayée, et aussi la brutalité de ses insultes, son obsession de la «nunnery », ses plaisanteries au moment où l'on va jouer le « théâtre dans le théâtre ».

En résumé : c'est la prise de conscience par Shakespeare de ce que peut avoir d'abstrait, d'irréel, même de mensonger, une certaine façon d'écrire la poésie qui a causé la nouvelle époque de son théâtre. Après avoir publié, avec quelque soin et un grand succès, Venus and Adonis puis le plus ambitieux The Rape of Lucrece, a-t-il été impressionné par le prestige des vers en leur environnement de subtil "limning", de jolie musique de cour, de belles manières, a-t-il considéré d'à tout le moins redoubler d'une réputation de poète celle que lui valait un genre, le théâtre, longtemps tenu pour mineur et que Sidney méprisait encore, en tout cas il a vite donné le pas sur ce moment de faiblesse à son besoin de comprendre. Et aux dépens de nouveaux poèmes il a choisi de réaffirmer la valeur de la scène, où les voix les plus diverses se croisent, et peuvent se contredire. Il y a un choix du théâtre, chez Shakespeare. Le choix d'y chercher la vraie poésie en un temps où la pratique des vers en était autant que jamais dans ce sonnet à la mode la contrefaçon ou, pour dire mieux, et plus généreusement, l'inconsciente méconnaissance.

Et combien avait-il raison, dans ces années qui n'étaient pas que celles des zélateurs du sonnet! Cette fin du $\mathrm{XvI}^{\mathrm{e}}$ siècle, c'est aussi la puissance préoccupante des puritains les plus extrémistes. Les hommes noirs dénoncent en toute femme la "dark lady", le religieux dégradé fait pendant à un trop facile lyrisme mais avec un pouvoir certes bien plus grand, qui risque de priver de théâtre la société apeurée. Comment résister au péril sinon en concentrant cette mémoire du vif qu'est la poésie dans le lieu de sa plus grande efficace, la scène théâtrale, et en allant là au plus vite à la vérité la plus forte? Autrement dit: faire du schème qu'ont révélé les sonnets - l'homme captif de sa vaine image, 
empêché de vivre, la femme vilipendée, la parole réduite à des lieux communs, le monde à de la chimère - l'instrument qui pénètre des situations que le dramaturge alors va exposer sur les planches, pour que chacun y reconnaisse son temps, s'y reconnaisse luimême, et s'alarme ou reprenne espoir. Rien n'est jamais tout à fait perdu, l'évidence simple de la vie déjoue parfois les pièges des idéologies les mieux établies dans la parole, c'est en tout cas la leçon qui ressort de ce que Shakespeare va écrire une fois sa décision prise, traversant d'abord le plus sombre, Macbeth, King Lear, Othello, mais proposant pour finir de méditer la résurrection d'Hermione du simple fait de l'intrépidité généreuse de sa fille perdue et retrouvée.

\section{AUTEUR}

\section{YVES BONNEFOY}

Yves Bonnefoy est professeur au Collège de France. Poète, il est également traducteur de Shakespeare auquel il a consacré de nombreux essais, récemment rassemblés en anglais dans Shakespeare and the French Poet, éd. John Naughton (Chicago U.P., 2004). 